Article

\title{
Two-Dimensional Correlation Function of Binary Black Hole Coalescences
}

\author{
Marco Cavaglià ${ }^{1, *(1)}$ and Ashini Modi ${ }^{2}$ \\ 1 Institute of Multi-messenger Astrophysics and Cosmology, Missouri University of Science and Technology, \\ Physics Building, 1315 N. Pine St., Rolla, MO 65409, USA \\ 2 Caddo Parish Magnet High School, 1601 Viking Dr., Shreveport, LA 71101, USA; modiashini2004@gmail.com \\ * Correspondence: cavagliam@mst.edu
}

Received: 20 May 2020; Accepted: 3 July 2020; Published: 7 July 2020

check for updates

\begin{abstract}
We compute the two-dimensional correlation functions of the binary black hole coalescence detections in LIGO-Virgo's first and second observation runs. The sky distribution of binary black hole coalescence events is tested for correlations at different angular scales by comparing the observed correlation function to two reference functions that are obtained from mock datasets of localization error regions uniformly distributed in the sky. No excess correlation at any angular scale is found. The power-law slope of the correlation function is estimated to be $\gamma=2.24 \pm 0.33$ at the three- $\sigma$ confidence level, a value consistent with the measured distribution of galaxies.
\end{abstract}

Keywords: gravitational waves; Universe large-scale structure; black holes

\section{Introduction}

On 14 September 2015, researchers from the Laser Interferometer Gravitational-wave Observatory (LIGO) [1] Scientific Collaboration (LSC) and the European Virgo Collaboration [2] made the first direct detection of Gravitational Waves (GWs) from a pair of coalescing black holes [3]. Less than two years after that first announcement, LIGO and Virgo observed GWs from the merger of two neutron stars [4], an event that was rapidly followed by the Fermi Gamma-ray Space Telescope's detection of a gamma-ray flash, and eventually by optical, infrared, radio, and X-ray observations by hundreds of telescopes around the world in what became the most observed event in the history of modern astronomy [5].

Currently, GW astronomy is a well-established scientific discipline. In the first two observing runs of Advanced LIGO and Virgo, O1 and O2, LSC and Virgo collaboration researchers observed ten Binary Black Hole $(\mathrm{BBH})$ coalescence detections and one binary neutron star coalescence detection. The third observation run, O3, brought us candidate detections on a weekly basis [6], enabling a plethora of novel astrophysical and theoretical investigations.

The next decade will see GW astronomy further expand its reach in frontier scientific research. Japan's KAGRA detector [7] has joined the international network of GW ground-based observatories. India has established the LIGO-India Scientific Collaboration (LISC) and finalized plans for the construction of the LIGO-India detector [8]. The European space-based LISA mission [9], slated to launch in 2034, will greatly improve detection capabilities and localizations of astrophysical sources. The International Pulsar Timing Array project will detect ultra-low frequency GWs within ten years [10]. Optical, particle, and GW astronomy will together explore the Universe through complementary physical carriers.

The publication of LIGO-Virgo's first catalog of compact binary merger signals [11] has shown that GW astrophysics is a powerful tool for population and source property studies of compact objects, tests of General Relativity, and large-scale cosmological measurements. However, many open 
questions still remain. For example, tests of GR have returned a null result [12]; the formation channels of black hole binaries [13] and the physics of EM-bright mergers [14] are still unclear, as well as the determination of the Hubble constant from GW sirens [15]. Despite LIGO and Virgo running all-sky, unmodeled searches [16,17], no GW signal has been detected that cannot be modeled as a compact binary coalescence. Other sources of multi-messenger signals such as isolated compact objects [18], core-collapse supernovae [19], and magnetars [20] have not been observed in the GW domain.

The rapid growth in the number of $\mathrm{BBH}$ coalescence detections and the dramatic improvement in their sky localizations are turning GW astrophysics into a precision observational science like large-scale structure astrophysics and early-Universe cosmology. One important physical concept in large-scale structure investigations and observational cosmology is that of the Correlation Function (CF) [21]. The (two-point) (auto-)CF describes the excess probability of finding pairs of points at a given separation. In large-scale astrophysics and observational cosmology, the CF (or its homolog in the frequency space, the power spectrum) is commonly used to describe the spatial distribution of galaxies or the density fluctuations observed in the cosmic microwave background. The CF from galaxy surveys, for example, allows astronomers to estimate the distance scales of galaxy clustering and gain information about the origin and evolution of the Universe's large-scale structures.

The purpose of this short article is to introduce the concept of the CF for BBH coalescence events. We use the public BBH coalescence detections in LIGO-Virgo's O1 and O2 runs to compute the two-dimensional $\mathrm{CF}$ for the population of these objects. Ten detections with sky localizations ranging from 39 square degrees to 1666 square degrees are clearly not sufficient to draw any meaningful conclusion on the spatial distribution of BBH coalescences. However, this calculation shows that the $\mathrm{CF}$ can be used to investigate the statistical properties of the population of these objects. We illustrate the method by comparing the two-dimensional CF obtained from the LIGO-Virgo O1-O2 BBH detections to a $\mathrm{CF}$ obtained by a random distribution of the same detections. The result shows that the two-dimensional spatial distribution of the detections is consistent with an isotropic distribution, as reported in Ref. [22] by implementing a pixelization-based method for the O1-O2 BBH detections. We also confirm this conclusion by comparing the $\mathrm{CF}$ to a synthetic $\mathrm{CF}$ obtained by simulating a number of $\mathrm{BBH}$ detections with sky localization error regions consistent with those of the LIGO-Virgo sample.

\section{Two-Dimensional Correlation Function}

In our analysis, we follow the customary definition for the two-dimensional (angular) CF of large-scale astrophysics [21]. The two-dimensional CF of a population of objects describes the excess probability of finding two objects separated by the angular distance $\theta$ with respect to a uniform distribution. To compute the $\mathrm{CF}$ of the $\mathrm{BBH}$ population, we treat the sky localization error regions of the $\mathrm{BBH}$ detections as probability density heat maps. Given the (normalized) sky localization error region map of the $i^{\text {th }} \mathrm{BBH}$ detection in the sample, $M_{i}(\chi, \varphi)$, where $\chi$ and $\varphi$ are the polar and azimuthal angles on the celestial sphere, respectively, we define the sky localization probability density map of the sample as:

$$
M(\chi, \varphi)=\frac{1}{A(N)} \sum_{i=1}^{N} F_{i}(\chi, \varphi) M_{i}(\chi, \varphi),
$$

where $N$ is the number of $\mathrm{BBH}$ detections, $F_{i}$ are probability weights that depend on the GW detector network sensitivity, and $A(N)$ is a normalization factor. By expanding the sky localization map in spherical harmonics,

$$
M(\chi, \varphi)=\sum_{l m} a_{l m} Y_{l m}(\chi, \varphi)
$$

the sky correlation function of the BBH sample can be defined as:

$$
C(\theta)=\left\langle M\left(\hat{n}_{1}\right) \cdot M\left(\hat{n}_{2}\right)\right\rangle_{21},
$$


where the average is taken over the observed sky with angular separation $\theta$ held fixed. Using the addition theorem of spherical harmonics, the CF can be written as:

$$
C(\theta)=\frac{1}{4 \pi} \sum_{l} a_{l}^{2} P_{l}(\cos \theta),
$$

where $P_{l}(\cos \theta)$ denotes the Legendre polynomial of order $l$ and argument $\cos \theta$, and we have defined $a_{l}^{2}=\sum_{m}\left|a_{l m}\right|^{2}$. Note that the CF in Equation (4) differs with the usual definition of the angular power spectrum that is used in Cosmic Microwave Background (CMB) cosmology, where $a_{l}^{2}=(2 l+1) C_{l}$. As the map $M(\chi, \varphi)$ describes a probability density field, rather than the perturbation field of a physical quantity, in the following, we focus on the CF instead of the power spectrum, which is the standard measure for fluctuation fields.

The quantities $a_{l}^{2}$ are measured from the sky localization map $M(\chi, \varphi)$ and determine the two-dimensional angular distribution of the BBH sample. Comparison of the $\mathrm{CF}$ to theoretical models involves modification of Equation (4) by multiplying the $a_{l}^{2}$ coefficients by a window function $W_{l}$ to take into account experimental constraints in the observations. For example, the finite beam resolution of the detector introduces a high- $l$ cutoff that can be modeled with a window function $W_{l} \propto \exp \left[-l(l+1) \sigma^{2}\right]$, where $\sigma$ is the detector resolution [21]. If the object population cannot be observed across the full sky, a mask is required. In contrast with CMB observations, where the region of the sky along the galactic plane must be masked in $\mathrm{CMB}$ observations due to the impossibility of measuring temperature fluctuations along the galactic plane, the full sky is transparent to GWs, and no mask is necessary. As the sky map in Equation (1) is obtained by summing the sky localization error regions of the $\mathrm{BBH}$ detections, the angular resolution is determined by the diffraction-limited spot size of the LIGO detectors:

$$
\theta_{\text {res }}=\frac{c}{2 d f},
$$

where $d$ is the typical separation of the detectors in the network, $c$ is the speed of light, and $f$ is the frequency of the measurement. Assuming a typical frequency of $200 \mathrm{~Hz}$ for the detector sensitivity and a LIGO-Virgo detector distance $d \sim 7000 \mathrm{~km}$, a crude estimate of the minimum map angular resolution is $\theta_{\text {res }} \sim 3^{\circ}$, or $\sigma \sim 1 / 30$, implying a high- $l$ cut-off of $l_{\max } \sim 30$.

In the following analysis, for the sake of simplicity, we assume the probability weights in Equation (1) to be constant, i.e., we assume that the sensitivity of the LIGO-Virgo detector network does not depend on the sky position (see Ref. [22] for a more refined analysis and a discussion on the effects of detector sensitivity on isotropic test of GW detections). An additional, possible modification of Equation (4) is due to the different sensitivities of the GW detector network across the O1-O2 epochs and the varying number of detectors observing each BBH event in the sample. These systematics can be eliminated, at least partially, by comparing the observed $C F C_{o b s}(\theta)$ to a CF, which is computed from a set of $\mathcal{N}$ reference maps $M_{\text {ref, } k}(\chi, \varphi), k=1, \ldots \mathcal{N}$, obtained by uniformly distributing the observed BBH sky localization error regions in the sky. A more refined analysis could be performed by injecting a population of simulated BBH signals with a uniform angular distribution and then creating the reference map by recovering the sky localization error regions of these injections with the GW network in the same configuration as in the real case. While this procedure would produce a more rigorous $\mathrm{CF}$ estimate than the one considered here, we consider it beyond the scope of this paper due to the small sample of $\mathrm{BBH}$ detections and the illustrative purpose of our analysis. We plan to revisit this procedure in a future work.

\section{Results}

We used the public sky localizations of the O1-O2 LIGO-Virgo BBH detections from the GW Open Science Center [23] and the open source Healpy package [24] to compute the CF. The sky localization error regions of the BBH detections came with different resolutions. We first rescaled each map to an NSIDE resolution of 256, corresponding to a pixel angular resolution of $\theta_{\text {pix }} \sim 0.23^{\circ} \ll \theta_{\text {res }}$. 
Choosing different values of the map resolution affected the final results only by a few percent. We then created the map $M_{\mathrm{obs}}(\chi, \varphi)$ in Equation (1) by summing the sky localization error regions of each $\mathrm{BBH}$ event and normalizing to the number of detections, such as $\sum_{j} M_{\mathrm{obs}}\left(p_{j}\right)=1$, where $p_{j}$ denotes the $j^{\text {th }}$ pixel. A Mollweide representation of $M_{\mathrm{obs}}(\chi, \varphi)$ in Equation (1) is shown in Figure 1.

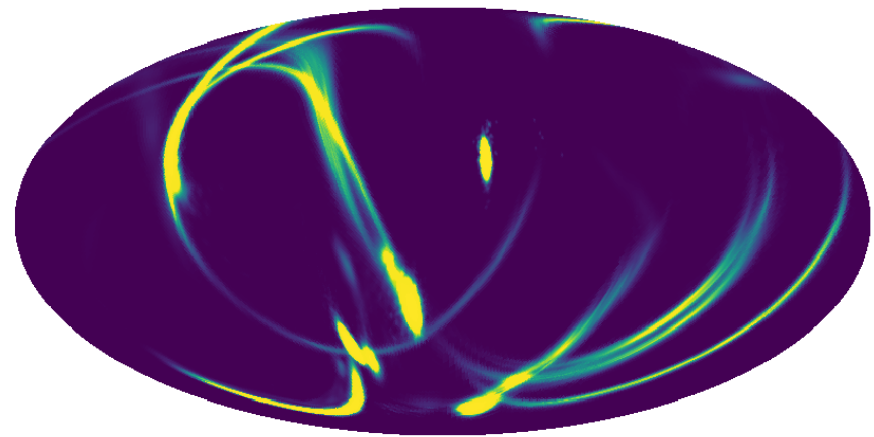

Figure 1. Heat sky map of the combined O1-O2 LIGO-Virgo detections, $M_{\mathrm{obs}}(\chi, \varphi)$. The color scale denotes the probability density of sky localization (yellow to blue: high to low, normalized to $\sum_{j} M\left(p_{j}\right)=0.1$ for better visualization purposes).

The $M_{\mathrm{obs}}(\chi, \varphi)$ map was treated as a heat map, and the Healpy function map2alm was used to compute $a_{l m}$. The coefficients of the Legendre expansion in Equation (4) were then obtained by summing the $\left|a_{l m}\right|^{2}$ in $m$. We followed the same procedure to compute the CF from reference maps $M_{\text {ref, } k}(\chi, \varphi)$ used to test possible angular correlation signatures in the CF.

In our analysis, we compared the observed $\mathrm{CF} C_{\mathrm{obs}}(\theta)$ to two reference $\mathrm{CFs}$. The first $\mathrm{CF}$ (Model A), $C_{\mathrm{ref}, A}(\theta)$, was obtained by averaging the CF of 500 artificial maps, each obtained by randomly rotating the maps of each single $\mathrm{BBH}$ detection in the sky by arbitrary $\chi$ and $\varphi$ angles. The second CF (Model B), $C_{\text {ref, } B}(\theta)$, was obtained by averaging 500 synthetic maps, each consisting of 10 elliptically-shaped sky localization error regions with random orientation and uniformly distributed in the sky. The sky localization areas of these artificial events were chosen such that their semi-axes were $R \cdot(x, 1 / x)$, where $x$ is uniformly distributed in $(0,10)$, and their area $\pi R^{2}$ was drawn from a lognormal distribution with mean (standard deviation) equal to the mean (standard deviation) of the sky localization areas of the observed events. Probability distribution contours of each of these artificial sky localization areas were simulated by superimposing 100 regions built as described above and radius decreasing as $f_{n}(R)=R \ln (2) / \ln (2+n)$, where $n=0 \ldots 99$. The above parameters reproduced sky localization error regions qualitatively similar to the observed $\mathrm{BBH}$ error regions while their variations did not significantly affect the final CF. Both reference maps were normalized to the number of detections in the sample, following the same procedure used for the observed map. One example of a synthetic map is shown in Figure 2.

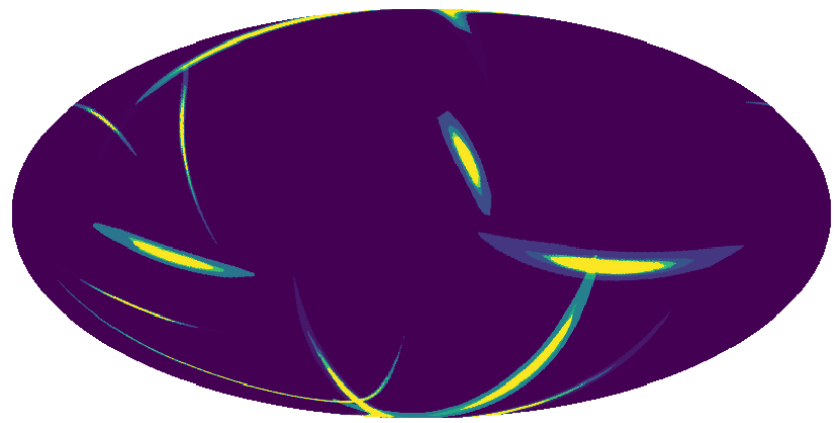

Figure 2. Example of a synthetic sky map for Model B. The (arbitrary) color scale denotes the probability density of sky localization (yellow to blue: high to low, normalized to $\sum_{j} M\left(p_{j}\right)=0.1$ for better visualization purposes). 
Figure 3 shows the observed CF compared to the reference $C F$ for Model A. $C_{\mathrm{obs}}(\theta)$ is represented in the top plot by the red curve. The five grey-shaded bands denote one through five standard deviations from the average of the CF computed on the reference maps $M_{\text {ref, } k}(\chi, \varphi)$ from Model A, $C_{\mathrm{ref}, A}(\theta)$. The bottom plot shows $C_{\mathrm{obs}}(\theta)$ normalized to $C_{\mathrm{ref}, A}(\theta)$. The observed $\mathrm{CF}$ lies entirely in the two- $\sigma$ band of the reference map, thus showing no excess correlation at any angular scale with respect to a uniform sky distribution of the O1-O2 detections. No excess correlation at any angular scale could be found when comparing $C_{\mathrm{obs}}(\theta)$ to the reference $C F$ for Model B, $C_{\text {ref, } B}(\theta)$. Results for this model are shown in Figure 4. The observed CF lied entirely within the 2- $\sigma$ error band of $C_{\text {ref, } B}(\theta)$. At small angular scales, $C_{\mathrm{obs}}(\theta)$ showed a lack of correlation compared to $C_{\mathrm{ref}, B}(\theta)$. This mismatch was likely due to the crude approximation used to simulate the artificial maps. As can be seen from Figure 1, the LIGO-Virgo sky localization error regions of observed BBH events are not perfect ellipses. Even if they were, their ellipticity would not follow a uniform distribution in their semi-axis ratio. Finally, drawing samples from a lognormal distribution of sky localization areas did not accurately represent the observed distribution of sky localizations in $\mathrm{O} 1$ and $\mathrm{O} 2$. A much more accurate estimate of BBH events' angular correlations could be obtained by simulating realistic sky maps by injecting, recovering, and localizing events according to the actual sensitivity of the GW detector network.
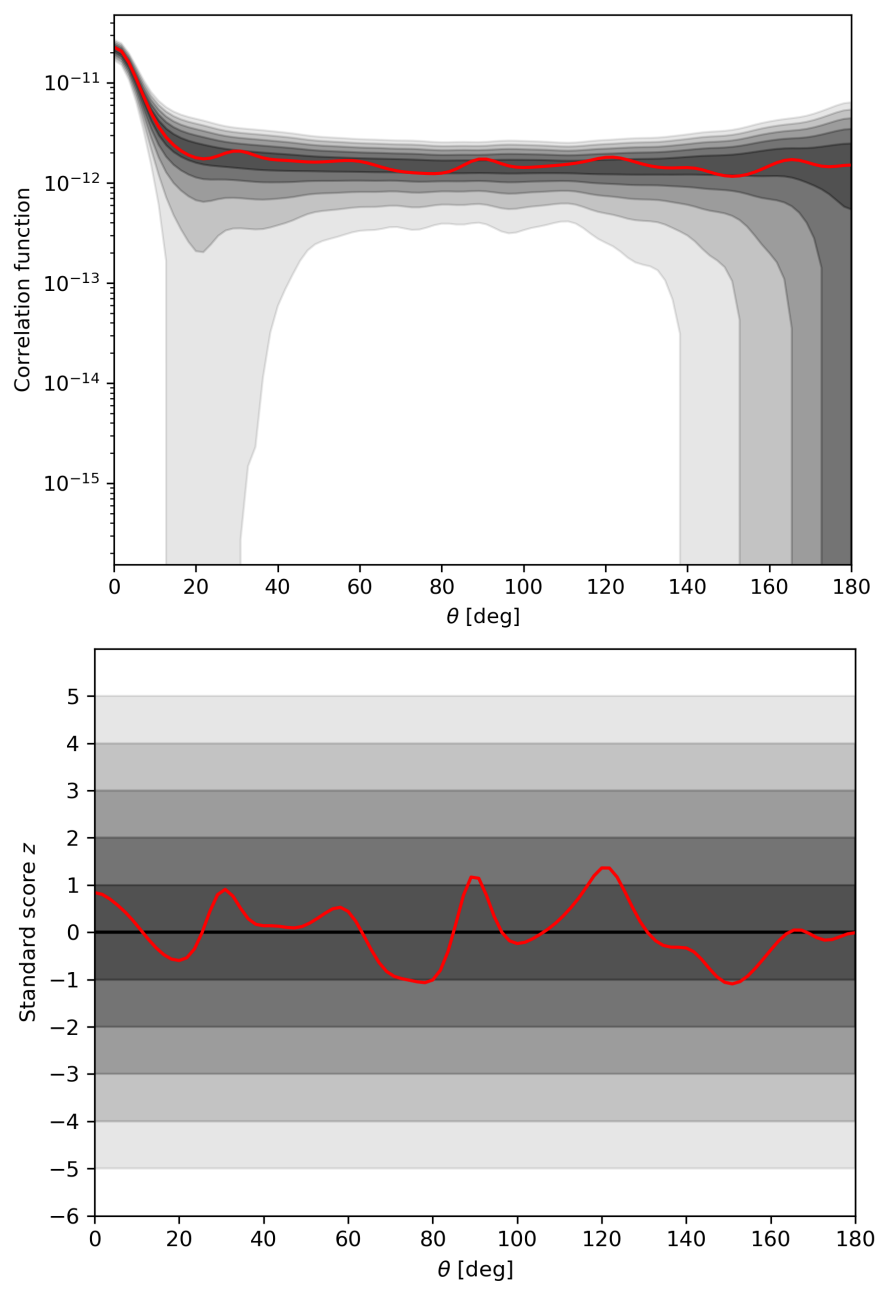

Figure 3. Top: Comparison of the measured CF (red continuous curve) and the reference CF for a set of maps obtained by randomly distributing the LIGO-Virgo observations in the sky (Model A). The grey-shaded bands denote one- through five- $\sigma$ deviations from the reference CF obtained by averaging over 500 "random" maps. Bottom: The observed CF normalized to the reference CF. The observed CF lies within two- $\sigma$ of $C_{\text {ref, } A}(\theta)$. 

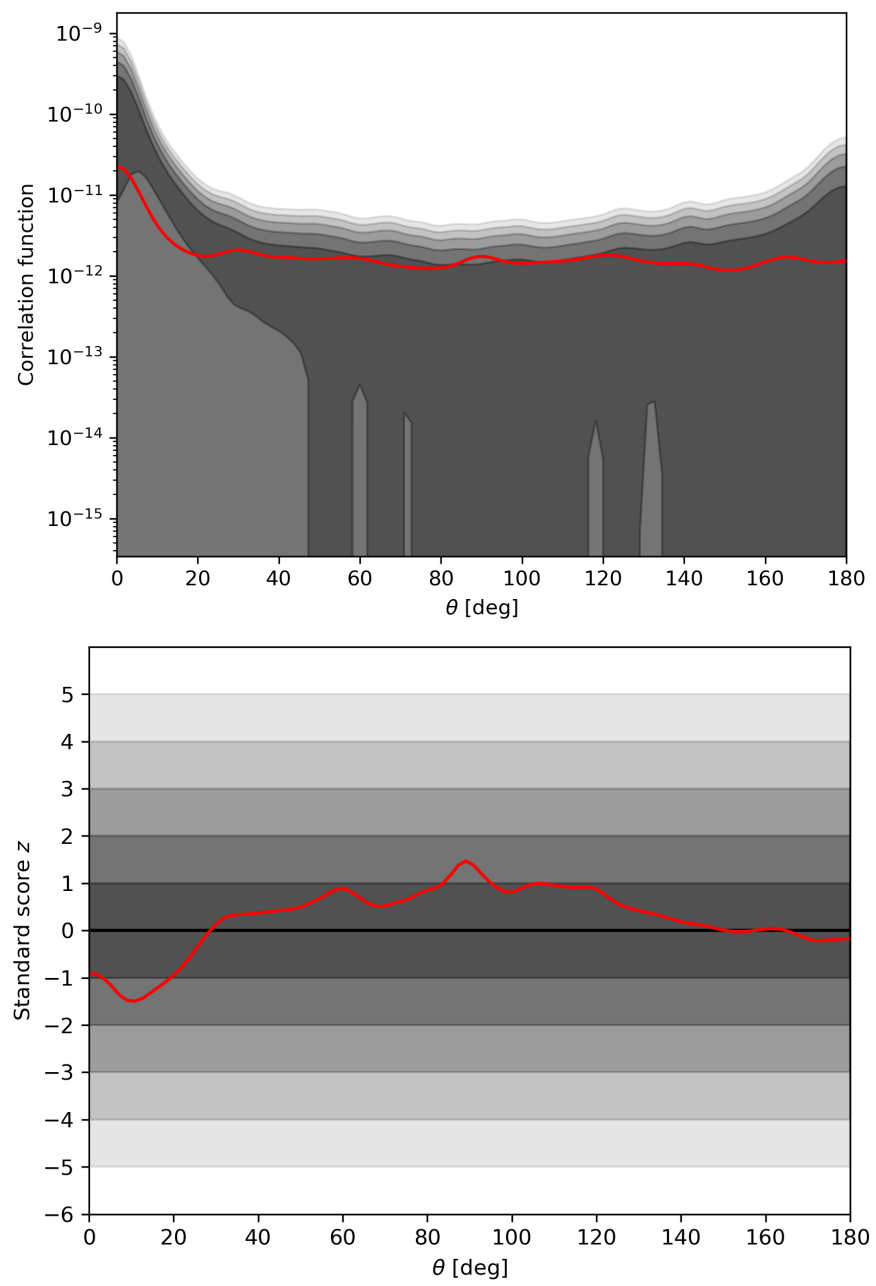

Figure 4. Top: Comparison of the measured CF (red continuous curve) and the CF for the set of synthetic maps of Model B. The grey-shaded bands denote one- through five- $\sigma$ deviations from the $\mathrm{CF}$ averaged over 500 synthetic maps. Bottom: Observed $z$-score of the observed CF for Model B. The observed $\mathrm{CF}$ lies within two- $\sigma$ of $C_{\mathrm{ref}, B}(\theta)$. The deviation at low angular distances is likely due to the approximation used to simulate the synthetic maps.

The CF in Equation (4) can be interpreted as a weighted projection of the spatial two point CF $\xi(r)$. At small scales, the power-law behavior of the CF is expected to be:

$$
C(\theta)=\left(\frac{\theta}{\theta_{0}}\right)^{1-\gamma}
$$

where $\theta_{0}$ is an angular correlation scale and $\gamma$ is the power-law slope of the spatial two point CF:

$$
\xi(r)=\left(\frac{r}{r_{0}}\right)^{-\gamma}
$$

where $r_{0}$ is the spatial correlation length. The power-law slope of the BBH distribution can be obtained by fitting $C_{\mathrm{obs}}(\theta)$ at small angular scales. A weighted best fit of Equation (6) from $\theta \sim \theta_{\text {res }}$ to $\theta \sim 18^{\circ}$, where departures from the power-law behavior become evident, gives for the power-law slope $\gamma=2.24 \pm 0.33$ at three- $\sigma$ confidence level, a value consistent with a uniform distribution of objects, $\xi(r) \sim r^{-2}$. As a comparison, the power-law slope from Sloan Digital Sky Survey (SDSS) data is $\gamma \sim 1.8$ over the range $0.005^{\circ}-10^{\circ}$ [25]. The VIMOS Public Extragalactic Redshift Survey (VIPERS) reports $\gamma \sim 1.7-1.8$ for a broad range of galaxy luminosities and stellar masses in the redshift range 
$0.5<z<1.1$ [26]. The VIMOS-VLT Deep Survey observes a significant redshift evolution of the luminosity dependence of the power-law slope parameter with $\gamma$ steepening from $\gamma \sim 1.7$ at low redshift to $\gamma \sim 2.4$ for $z \sim 0.9$ and galaxies with high intrinsic luminosity [27]. In contrast to the SDSS, VIPERS, and VIMOS-VLT results, which point to galaxy clustering in the redshift range of BBH detections, $z \lesssim 0.5$, our result showed no evidence of clustering at these distance scales. It would be interesting to test whether any evidence of clustering would appear in the data with more BBH detections and better sample statistic.

\section{Conclusions}

In this short article, we computed the two-dimensional CF of BBH observations in the first and second observation runs of advanced LIGO and Virgo. The CF is commonly used in large-scale structure astrophysics and precision cosmology to quantify the spatial distribution of an object class population. Similarly, we used the two-dimensional CF to measure the statistical properties of the BBH coalescence spatial distribution. By comparing the CF of the LIGO-Virgo detections to a simulated $\mathrm{CF}$ from a synthetic sample of sky localizations and a $\mathrm{CF}$ obtained by randomly re-orienting the $\mathrm{BBH}$ detections, we showed that the distribution of O1-O2 BBH events in the sky was in agreement with a uniform distribution of sources, as previously reported in Ref. [22]. The power-law slope of the CF was found to be $\gamma=2.24 \pm 0.33$, a value consistent with the upper bound of the power-law slope from galaxy surveys at low redshift $z$.

While the limited number of O1-O2 detections with large sky localization error regions did not allow us to draw any significant physical conclusions, our work lays the formalism for computing the CF of a class of GW detections. Our analysis was clearly rudimentary and could be improved in many ways. The extension to the tens of LIGO-Virgo detections in $\mathrm{O} 3$ is straightforward. A better estimate for the two-dimensional spatial distribution of $\mathrm{BBH}$ coalescence events could be obtained by comparing the detected CF to a synthetic CF from a realistic population of events as done in Ref. [22]. This could be done by testing the detected CF against a CF from injection sets consistent with the observed $\mathrm{BBH}$ coalescence population and detector network sensitivity. The existence of angular correlations in the spatial distribution of BBH coalescences could be tested by building CFs for events distributed isotropically in the sky, or at given angular scales. Comparisons of the observed BBH CF to CFs of anisotropic models for the astrophysical GW background [28] and other astrophysical objects could be used to test correlations of BBH events with the spatial distribution of these objects [29], test BBH population paradigms, and probe fundamental physics [30,31]. Our method could also be extended to include information about the distances of the BBH sources by computing the three-dimensional CF [32]. The latter could be compared to CFs obtained from given models of population synthesis, as well as three-dimensional CFs of other astrophysical objects. With the anticipated higher rate of detections and more accurate sky localizations in future LIGO-Virgo observing runs, the CF of BBH and other GW-bright sources may prove itself as another useful tool for GW astronomy investigations.

Author Contributions: All authors have contributed equally to all aspects of this work. All authors have read and agreed to the published version of the manuscript.

Funding: This research was funded by the U.S. National Science Foundation grant number PHY-1921006.

Acknowledgments: This work started as a high-school science project of A.M. at Caddo Parish Magnet High School in Shreveport, LA, under the mentorship of M.C. It was first presented at the LA state science fair in fall 2019. For this project, A.M. was awarded the Association for Women Geoscientists award, the U.S. Agency for International Development (USAID) award, the Office of Naval Research/U.S. Navy/U.S. Marine Corps award, and the NASA Earth System Science Award. A.M. thanks the teachers and mentors at the Caddo Parish Magnet High School for their continuous support. M.C. is supported by the U.S. National Science Foundation grant PHY-1921006. LIGO was constructed and is operated by the California Institute of Technology and Massachusetts Institute of Technology with funding from the U.S. National Science Foundation under grant PHY-0757058. Virgo is funded by the French Centre National de la Recherche Scientifique (CNRS), the Italian Istituto Nazionale di Fisica Nucleare (INFN), and the Dutch Nikhef, with contributions by Polish and Hungarian institutes. The authors would like to thank their LIGO Scientific Collaboration and Virgo Collaboration colleagues for their help and useful comments, in particular Vuk Mandic, Kentaro Mogushi, Ryan Quitzow-James, Joe Romano, and Mairi 
Sakellariadou. The authors are grateful for computational resources provided by the LIGO Laboratory and supported by the U.S. National Science Foundation grants PHY-0757058 and PHY-0823459, as well as resources from the Gravitational Wave Open Science Center, a service of LIGO Laboratory, the LIGO Scientific Collaboration, and the Virgo Collaboration. This research made use of data, software, and web tools obtained from the Gravitational Wave Open Science Center and openly available at https:/ / www.gw-openscience.org. Healpy is licensed under the GNU General Public License. This manuscript has been assigned LIGO Document Control Center Number LIGO-P2000157.

Conflicts of Interest: The authors declare no conflict of interest.

\section{References}

1. Aasi, J.; et al. [LIGO Scientific Collaboration]. Advanced LIGO. Class. Quantum Gravity 2015, $32,074001$. [CrossRef]

2. Acernese, F. [Virgo Collaboration]. The Advanced Virgo detector. J. Phys. Conf. Ser. 2015, 610, 012014. [CrossRef]

3. Abbott, B.P.; et al. [LIGO Scientific and Virgo Collaborations]. Observation of Gravitational Waves from a Binary Black Hole Merger. Phys. Rev. Lett. 2016, 116, 061102. [CrossRef] [PubMed]

4. Abbott, B.P.; et al. [LIGO Scientific and Virgo Collaborations]. Phys. Rev. Lett. 2017, 119, 161101. [CrossRef]

5. Abbott, B.P.; et al. [LIGO Scientific and Virgo and Fermi GBM and INTEGRAL and IceCube and IPN and Insight-Hxmt and ANTARES and Swift and Dark Energy Camera GW-EM and DES and DLT40 and GRAWITA and Fermi-LAT and ATCA and ASKAP and OzGrav and DWF (Deeper Wider Faster Program) and AST3 and CAASTRO and VINROUGE and MASTER and J-GEM and GROWTH and JAGWAR and CaltechNRAO and TTU-NRAO and NuSTAR and Pan-STARRS and KU and Nordic Optical Telescope and ePESSTO and GROND and Texas Tech University and TOROS and BOOTES and MWA and CALET and IKI-GW Follow-up and H.E.S.S. and LOFAR and LWA and HAWC and Pierre Auger and ALMA and Pi of Sky and DFN and ATLAS Telescopes and High Time Resolution Universe Survey and RIMAS and RATIR and SKA South Africa/MeerKAT Collaborations and AstroSat Cadmium Zinc Telluride Imager Team and AGILE Team and 1M2H Team and Las Cumbres Observatory Group and MAXI Team and TZAC Consortium and SALT Group and Euro VLBI Team and Chandra Team at McGill University]. Multi-messenger Observations of a Binary Neutron Star Merger. Astrophys. J. 2017, 848, L12. [CrossRef]

6. GraceDB-The Gravitational-wave Candidate Event Database. Available online: http://gracedb.ligo.org (accessed on 20 May 2020).

7. Akutsu, T.; et al. [KAGRA Collaboration]. KAGRA: 2.5 Generation Interferometric Gravitational Wave Detector. Nat. Astron. 2019, 3, 35. [CrossRef]

8. The Laser Interferometer Gravitational-Wave Observatory (LIGO)-India. Available online: http://www. ligo-india.in (accessed on 20 May 2020).

9. LISA: Laser Interferometer Space Antenna. A Proposal in Response to the ESA Call for L3 Mission Concepts. Available online: https://www.elisascience.org/files/publications/LISA_L3_20170120.pdf (accessed on 20 May 2020).

10. Hobbs, G.; Archibald, A.; Arzoumanian, Z.; Backer, D.; Bailes, M.; Bhat, N.D.R.; Burgay, M.; Burke-Spolaor, S.; Champion, D.; Cognard, I.; et al. The international pulsar timing array project: Using pulsars as a gravitational wave detector. Class. Quantum Gravity 2010, 27, 084013. [CrossRef]

11. Abbott, B.P.; et al. [LIGO Scientific and Virgo Collaborations]. GWTC-1: A Gravitational-Wave Transient Catalog of Compact Binary Mergers Observed by LIGO and Virgo during the First and Second Observing Runs. Phys. Rev. X 2019, 9, 031040. [CrossRef]

12. Abbott, B.P.; et al. [LIGO Scientific and Virgo Collaborations]. Tests of General Relativity with the Binary Black Hole Signals from the LIGO-Virgo Catalog GWTC-1. arXiv 2019, arXiv1903.04467.

13. Abbott, B.P.; et al. [LIGO Scientific and Virgo Collaborations]. Binary Black Hole Population Properties Inferred from the First and Second Observing Runs of Advanced LIGO and Advanced Virgo. Astrophys. J. 2019, 882, L24. [CrossRef]

14. Abbott, B.P.; et al. [LIGO Scientific and Virgo Collaborations]. Model comparison from LIGO-Virgo data on GW170817's binary components and consequences for the merger remnant. arXiv 2019, arXiv:1908.01012. 
15. Abbott, B.P.; et al. [LIGO Scientific and Virgo Collaborations]. A gravitational-wave measurement of the Hubble constant following the second observing run of Advanced LIGO and Virgo. arXiv 2019, arXiv:1908.06060.

16. Abbott, B.P.; et al. [LIGO Scientific and Virgo Collaborations]. All-sky search for short gravitational-wave bursts in the second Advanced LIGO and Advanced Virgo run. Phys. Rev. D 2019, 100, 024017. [CrossRef]

17. Abbott, B.P.; et al. [LIGO Scientific and Virgo Collaborations]. All-sky search for long-duration gravitational-wave transients in the second Advanced LIGO observing run. Phys. Rev. D 2019, 99, 104033. [CrossRef]

18. Abbott, B.P.; et al. [LIGO Scientific and Virgo Collaborations]. All-sky search for continuous gravitational waves from isolated neutron stars using Advanced LIGO O2 data. Phys. Rev. D 2019, 100, 024004. [CrossRef]

19. Abbott, B.P.; et al. [LIGO Scientific and Virgo Collaborations]. An Optically Targeted Search for Gravitational Waves emitted by Core-Collapse Supernovae during the First and Second Observing Runs of Advanced LIGO and Advanced Virgo. arXiv 2019, arXiv:1908.03584.

20. Abbott, B.P.; et al. [LIGO Scientific and Virgo Collaborations]. Search for Transient Gravitational-wave Signals Associated with Magnetar Bursts during Advanced LIGO's Second Observing Run. Astrophys. J. 2019, 874, 163. [CrossRef]

21. White, M.; Scott, D.; Silk, J. Anisotropies in the Cosmic Microwave Background. Annu. Rev. Astron. Astrophys. 1994, 32, 319-370. [CrossRef]

22. Stiskalek, R.; Veitch, J.; Messenger, C. Are stellar mass binary black hole mergers isotropically distributed? arXiv 2020, arXiv:2003.02919.

23. Abbott, R.; et al. [LIGO Scientific and Virgo Collaborations]. Open data from the first and second observing runs of Advanced LIGO and Advanced Virgo. arXiv 2019, arXiv:1912.11716.

24. Healpy, a Python Package to Handle Pixelated Data on the Sphere. Available online: healpy.readthedocs.io/ en/latest.index.html (accessed on 20 May 2020).

25. Wang, Y.; Brunner, R.J.; Dolence, J.C. The SDSS Galaxy Angular Two-Point Correlation Function. Mon. Not. R. Astron. Soc. 2013, 432, 1961-1979. [CrossRef]

26. Marulli, F.; Bolzonella, M.; Branchini, E.; Davidzon, I.; De La Torre, S.; Granett, B.R.; Guzzo, L.; Iovino, A.; Moscardini, L.; Pollo, A.; et al. The VIMOS Public Extragalactic Redshift Survey (VIPERS)-Luminosity and stellar mass dependence of galaxy clustering at $0.5<z<1.1$. Astron. Astrophys. 2013, 557, A17.

27. Pollo, A.; Guzzo, L.; Fèvre, O.L.; Meneux, B.; Cappi, A.; Franzetti, P.; Iovino, A.; McCracken, H.J.; Marinoni, C.; Zamorani, G.; et al. The VIMOS-VLT Deep Survey-Luminosity dependence of clustering at $z \sim 1$. Astron. Astrophys. 2006, 451, 409. [CrossRef]

28. Jenkins, A.C.; Sakellariadou, M.; Regimbau, T.; Slezak, E. Anisotropies in the astrophysical gravitationalwave background: Predictions for the detection of compact binaries by LIGO and Virgo. Phys. Rev. D 2018, 98, 063501. [CrossRef]

29. Banagiri, S.; Mandic, V.; Scarlata, C.; Yang, K.Z. Measuring angular N-point correlations of binary black-hole merger gravitational-wave events with hierarchical Bayesian inference. arXiv 2020, arXiv:2006.00633.

30. Mukherjee, S.; Wandelt, B.D.; Silk, J. Multi-messenger tests of gravity with weakly lensed gravitational waves. Phys. Rev. D 2020, 101, 103509. [CrossRef]

31. Mukherjee, S.; Wandelt, B.D. Beyond the classical distance-redshift test: Cross-correlating redshift-free standard candles and sirens with redshift surveys. arXiv 2018, arXiv:1808.06615.

32. Vijaykumar, A.; Saketh, M.; Kumar, S.; Ajith, P.; Choudhury, T.R. Probing the large scale structure using gravitational-wave observations of binary black holes. arXiv 2020, arXiv:2005.01111.

(c) 2020 by the authors. Licensee MDPI, Basel, Switzerland. This article is an open access article distributed under the terms and conditions of the Creative Commons Attribution (CC BY) license (http://creativecommons.org/licenses/by/4.0/). 